\author{
P. V. Ramana ${ }^{1}$ B. S. Krishna ${ }^{1}$, N. B. Reddy ${ }^{1,2}$, \\ G. Sravaya ${ }^{1,2}$, G. V. Zyryanov ${ }^{2,3}$, C. S. Reddy ${ }^{1}$ \\ ${ }^{1}$ Department of Chemistry, Sri Venkateswara University, \\ Tirupati, 517502, India \\ Tel: + 91-9849694958; Fax: + 918772289555 \\ E-mail:csrsvu@gmail.com \\ ${ }^{2}$ Department of Organic and Biomolecular Chemistry, \\ Institute of Chemical Engineering, Ural Federal University, \\ 19 Mira St., Ekaterinburg, 620002, Russia \\ ${ }^{3}$ Postovsky Institute of Organic Synthesis UB RAS, \\ 22 Kovalevskaya St. / 20 Akademicheskaya St., \\ Ekaterinburg, 620990, Russia
}

\title{
Potassium carbonate supported efficient synthesis of new diethyl arylphosphoramidates
}

A series of some new diethyl arylphosphoramidates have been synthesized from the reaction of diethyl chlorophosphate with different amines in the presence of $5 \mathrm{~mol} \%$ of potassium carbonate catalyst. This reaction is operationally simple and efficient to afford the products with high yields in short reaction times. All the compounds synthesized were characterized by spectroscopic and elemental analysis. Summarizing our catalyst and solvent optimization studies we are reporting that potassium carbonate and DMSO is a best catalyst system for the synthesis of phosphoramidates.

Keywords: phosphoramidates, efficient synthesis, potassium carbonate catalyst, DMSO

Received: 14.06.2017; accepted: 27.06.2017; published: 14.07.2017.

(c) Ramana P. V., Krishna B. S., Reddy N. B., Sravaya G., Zyryanov G. V., Reddy C. S., 2017

\section{Introduction}

Phosphoramidates have gained considerable interest in the last few decades as they have various applications in organic synthesis such as catalytic conversions like aldol and allylation reactions [1]. In addition to catalytic applications, $\mathrm{N}$-arylphosphoramidates have been used as precursors for the synthesis of various heterocycles such as azetidines, aziridines, quinazolinediones and imines [2-3]. Beside this, they are also used to synthesize phosphate esters in nucleotides chemistry [4]. In analytical chemistry, phosphoramidates improve ionization ef- ficiency and suppress matrix-related ion effects in MALDITOF mass spectrometry [5]. In medicinal chemistry, it is reported that phosphoramidates can be used as prodrug moieties to improve therapeutic potential of the parent drug [6]. Phosphoramidates have also served as surrogates for amide bond in the synthesis of peptide based protease inhibitors [7]. They represents some key structure in a number of biologically active natural products like agrocin 84 [8], phosmidosine (II) [9] and GS-6620 (III) [10]. They also form important pharmacophore of many biologi- 
cally potent compounds e.g. sofosbuvir (IV) (FDA approved drug) used for the treatment of hepatitis $\mathrm{C}$ virus (HCV) [11], evofosfamidum (TH-302) (V) which is in clinical trials for cancer treatment (Fig. 1) [12]. Recently, phosphoramidates have also been used in the field of plant hormone as abscisic acid (ABA) agonists that play role in plant growth regulators [13].

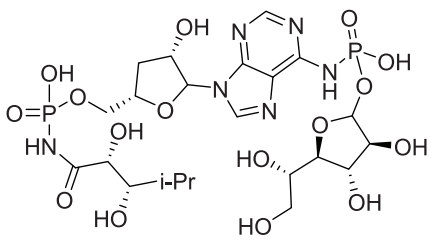

(I)

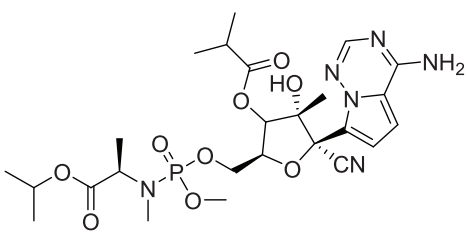

(III)

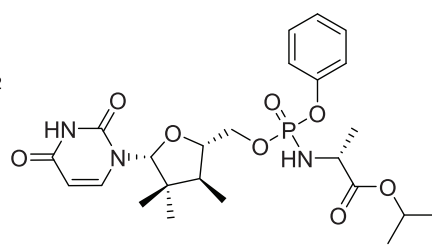

(IV)

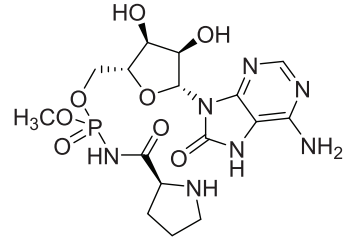

(II) reported with the similar reactivity as we expected in alkylations [15]. These phosphoramidates featuring a P-N bond are used as pesticides in agriculture and prodrugs in therapeutic development, and for other synthetic applications [16]. Furthermore, they have been utilized as ligands for metal-catalyzed organic transformations, as flame retardants, and as

Fig. 1. Some representative bioactive phosphoramidates

Among literature methods, direct phosphorylation of different amines with phosphorus halides is one of the most attractive and synthetically accessible methods [14]. Coming to the reactivity, $\mathrm{N}$-phosphorylation of the $\mathrm{NH}$ moiety of few $\mathrm{N}$-heterocycles like indoles, imidazoles, and benzimidazole derivatives was labelling groups to improve sensitivity in mass spectroscopy [17]. The phosphorylation of a series of amines was studied under different conditions involving the application of the various methods. Our aim was to find the best set of conditions for the preparation of some of these phosphoramidates.

\section{Experimental}

General: All reagents were obtained from Sigma-Aldrich and Alfa Aesar and were used directly without further purification. Melting points were recorded on Guna Digital Melting Point apparatus. IR spectra were recorded on Bruker Alpha Eco ATR - FTIR interferometer with single reflection sampling module equipped with $\mathrm{ZnSe}$ crystal. ${ }^{1} \mathrm{H},{ }^{13} \mathrm{C}$ and
${ }^{31} \mathrm{P}$ NMR spectra were recorded on Bruker AMX $500 \mathrm{MHz}$ NMR spectrometers operating at $400 \mathrm{MHz}$ for ${ }^{1} \mathrm{H}, 100 \mathrm{MHz}$ for ${ }^{13} \mathrm{C}$ and $160 \mathrm{MHz}$ for ${ }^{31} \mathrm{P}$ NMR in DMSO and were referenced to TMS $\left({ }^{1} \mathrm{H}\right.$ and $\left.{ }^{13} \mathrm{C}\right)$ and $85 \% \mathrm{H}_{3} \mathrm{PO}_{4}\left({ }^{31} \mathrm{P}\right)$ and their chemical shifts were reported in $\delta$ scale. Mass spectra were recorded on a Jeol SX 102 DA/600 mass spectrometer and elemental analy- 
sis was performed on a Thermo Finnigan Instrument. Melting points were determined in open capillaries using EZ-Melt automated melting point apparatus. All solvents used for spectroscopic and other physical studies were reagent grade and were further purified by methods reported in the literature.

Chemistry: Initially $0.127 \mathrm{mG}$ (1 mmol) of 4-Chloro aniline (1a) was added to $0.144 \mathrm{~mL}$ (1 $\mathrm{mmol}$ ) diethyl chlorophosphate (2) along with $\mathrm{K}_{2} \mathrm{CO}_{3}$ (5 mol\%) into a $50 \mathrm{~mL}$ round bottom flask in $8 \mathrm{~mL}$ of DMSO. Then it is equipped with a reflux condenser and the contents were heated to $80{ }^{\circ} \mathrm{C}$ and reaction was continued at the same temperature and the reaction progress was monitored with TLC (3:7 ratio of ethylacetate and hexane mixture). After completion of the reaction the crude contents of diethyl (4-chlorophenyl) phosphoramidate (3a) formed were cooled to room temperature and was cooled to room temperature conditions. Then the filtrate was concentrated by removing the solvent by rota-evaporation and then it was purified by column chromatography (1:9 ratio of ethylacetate and hexane mixture) and the pure product $3 \mathbf{a}$ was collected.

Similarly various amines amines (1a-n) as listed above were used to synthesize corresponding diethyl arylphosphoramidates (3a-n) with good reaction yields by the catalytic action of $\mathrm{K}_{2} \mathrm{CO}_{3}$ (5 mol \%) in DMSO at $80^{\circ} \mathrm{C}$ (Fig. 2).

Diethyl (4-chlorophenyl)phosphoramidate (3a): Yield: 92\%; Brown solid; IR (ZnSe): 3312 (NH Aromatic), 1172 $(\mathrm{P}=\mathrm{O}), 935\left(\mathrm{P}-\mathrm{O}-\mathrm{C}_{\text {aliphatic }}\right) \mathrm{cm}^{-1} ;{ }^{1} \mathrm{H} \mathrm{NMR}$ $\left(500 \mathrm{MHz}, \mathrm{DMSO}-d_{6}\right): \delta 1.17-1.33(6 \mathrm{H}$, $\left.\mathrm{m}, 2 \mathrm{CH}_{3}\right), 3.95(1 \mathrm{H}, \mathrm{s}, \mathrm{NH}), 4.26-4.34(4 \mathrm{H}$, m, 2CH 2 ), 7.06-7.72 (4H, m, ArH) ppm; ${ }^{13} \mathrm{C}$ NMR (125 MHz, DMSO- $\left.d_{6}\right): \delta 16.48$,
63.32, 119.26, 127.08, 129.54, 142.22 ppm; ${ }^{31} \mathrm{P}$ NMR (200 MHz, DMSO- $\left.d_{6}\right)$ : $\delta 2.856 \mathrm{ppm}$; LC-MS m/z (\%): 263 (100) $[\mathrm{M}+]$; Anal. Calcd. for $\mathrm{C}_{10} \mathrm{H}_{15} \mathrm{ClNO}_{3} \mathrm{P}$ (\%): C, 45.55; H, 5.73; N, 5.31. Found: C, 45.51, H, 5.69; N, 5.28.

\section{Diethyl (4-fluorophenyl)phospho-} ramidate (3b): Yield: 89\%; Brown solid; IR (ZnSe): 3325 ( $\mathrm{NH}$ Aromatic), 1225 $(\mathrm{P}=\mathrm{O}), 942\left(\mathrm{P}-\mathrm{O}-\mathrm{C}_{\text {aliphatic }}\right) \mathrm{cm}^{-1} ;{ }^{1} \mathrm{H} \mathrm{NMR}$ $\left(500 \mathrm{MHz}, \mathrm{DMSO}-d_{6}\right): \delta 1.20-1.28(6 \mathrm{H}$, $\left.\mathrm{m}, 2 \mathrm{CH}_{3}\right), 3.87(1 \mathrm{H}, \mathrm{s}, \mathrm{NH}), 4.22-4.26(4 \mathrm{H}$, $\left.\mathrm{m}, 2 \mathrm{CH}_{2}\right), 6.65-7.06(4 \mathrm{H}, \mathrm{m}, \mathrm{ArH}) \mathrm{ppm}$; ${ }^{13} \mathrm{C}$ NMR (125 MHz, DMSO- $\left.d_{6}\right): \delta 16.42$, 62.96, 118.52, 125.85, 132.34, 148.44 ppm; ${ }^{31} \mathrm{P}$ NMR (200 MHz, DMSO- $\left.d_{6}\right)$ : $\delta 2.824$ ppm; LC-MS m/z (\%): 247 (100) $[\mathrm{M}+]$; Anal. Calcd. for $\mathrm{C}_{10} \mathrm{H}_{15} \mathrm{ClNO}_{3} \mathrm{P}(\%)$ : C, 48.59; H, 6.12; N, 5.67. Found: C, 48.51; $\mathrm{H}, 6.06 ; \mathrm{N}, 5.63$.

Diethyl (4-methoxyphenyl)phosphoramidate (3c): Yield: $90 \%$; Brown solid; IR (ZnSe): 3321 ( $\mathrm{NH}$ Aromatic), 1212 $(\mathrm{P}=\mathrm{O}), 938$ (P-O-C $\mathrm{aliphatic}) \mathrm{cm}^{-1} ;{ }^{1} \mathrm{H}$ NMR $\left(500 \mathrm{MHz}, \mathrm{DMSO}-d_{6}\right): \delta 1.18-1.29(6 \mathrm{H}$, $\left.\mathrm{m}, 2 \mathrm{CH}_{3}\right), 3.78-3.84\left(3 \mathrm{H}, \mathrm{m},-\mathrm{O}-\mathrm{CH}_{3}\right)$, $3.89(1 \mathrm{H}, \mathrm{s}, \mathrm{NH}), 4.01-4.12(4 \mathrm{H}, \mathrm{m}$, $\left.2 \mathrm{CH}_{2}\right), 6.59-6.94(4 \mathrm{H}, \mathrm{m}, \mathrm{ArH}) \mathrm{ppm}$; ${ }^{13} \mathrm{C}$ NMR (125 MHz, DMSO- $\left.d_{6}\right): \delta 16.38$, 55.82, 62.96, 117.33, 121.48, 132.94, $152.31 \mathrm{ppm} ;{ }^{31} \mathrm{P}$ NMR (200 MHz, DMSO$\left.d_{6}\right): \delta 2.836 \mathrm{ppm} ; \mathrm{LC}-\mathrm{MS} m / z(\%): 259$ (100) $[\mathrm{M}+]$; Anal. Calcd. for $\mathrm{C}_{11} \mathrm{H}_{18} \mathrm{NO}_{4} \mathrm{P}$ (\%): C, 50.96; H, 7.00; N, 5.40. Found: C, $50.92 ; \mathrm{H}, 6.95 ; \mathrm{N}, 5.33$.

\section{Diethyl (5-nitropyridin-2-yl)phos-} phoramidate (3d): Yield: $90 \%$; Brown solid; IR (ZnSe): 3332 ( $\mathrm{NH}$ Aromatic), $1194(\mathrm{P}=\mathrm{O}), 945\left(\mathrm{P}-\mathrm{O}-\mathrm{C}_{\text {aliphatic }}\right) \mathrm{cm}^{-1} ;{ }^{1} \mathrm{H}$ NMR (500 MHz, DMSO-d $): \delta 1.24-1.31$ $\left(6 \mathrm{H}, \mathrm{m}, 2 \mathrm{CH}_{3}\right), 3.98(1 \mathrm{H}, \mathrm{s}, \mathrm{NH}), 4.35-$ $4.46\left(4 \mathrm{H}, \mathrm{m}, 2 \mathrm{CH}_{2}\right), 7.06-8.72(4 \mathrm{H}, \mathrm{m}$, ArH) ppm; ${ }^{13} \mathrm{C}$ NMR (125 MHz, DMSO$\left.d_{6}\right): \delta 16.36,62.21,110.96,132.08,136.22$, 
144.78, 169.14 ppm; ${ }^{31} \mathrm{P}$ NMR $(200 \mathrm{MHz}$, DMSO- $\left.d_{6}\right): \delta 2.842 \mathrm{ppm}$; LC-MS $\mathrm{m} / z$ (\%): 275 (100) $[\mathrm{M}+]$; Anal. Calcd. for $\mathrm{C}_{9} \mathrm{H}_{14} \mathrm{~N}_{3} \mathrm{O}_{5} \mathrm{P}$ (\%): C, 39.28; $\mathrm{H}, 5.13 ; \mathrm{N}$, 15.27. Found: C, 39.24; H, 5.09; N, 15.21 .
Diethyl

(3-fluoro-5-nitrophenyl) phosphoramidate (3e): Yield: 92\%; Brown solid; IR (ZnSe): 3348 (NH Aromatic), $1209(\mathrm{P}=\mathrm{O}), 944 \quad\left(\mathrm{P}-\mathrm{O}-\mathrm{C}_{\text {aliphatic }}\right)$ $\mathrm{cm}^{-1}$; ${ }^{1} \mathrm{H}$ NMR (500 MHz, DMSO-d ${ }_{6}$ ):

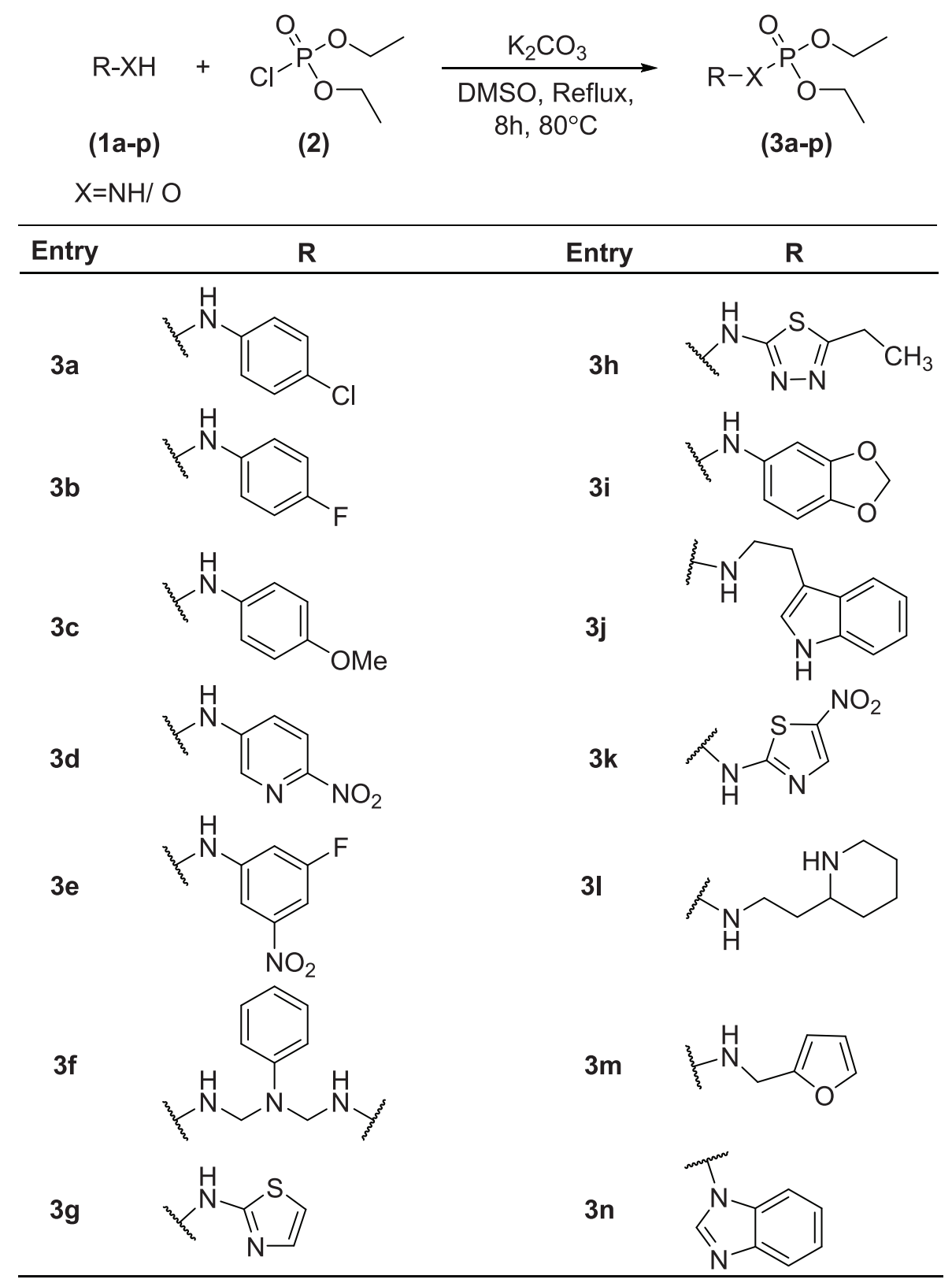

Fig. 2. Synthesis of diethyl arylphosphoramidates 
$\delta$ 1.19-1.32 $\left(6 \mathrm{H}, \mathrm{m}, 2 \mathrm{CH}_{3}\right), 4.02(1 \mathrm{H}, \mathrm{s}$, $\mathrm{NH}), 4.42-4.48$ (4H, m, 2CH $), 7.02-7.42$ $(3 \mathrm{H}, \mathrm{m}, \mathrm{ArH}) \mathrm{ppm} ;{ }^{13} \mathrm{C} \mathrm{NMR}(125 \mathrm{MHz}$, DMSO- $\left.d_{6}\right): \delta 16.08,62.04,102.05,104.54$, 111.22, 142.08, 150.65, 165.84 ppm; ${ }^{31} \mathrm{P}$ NMR (200 MHz, DMSO- $\left.d_{6}\right): \delta 2.816$ ppm; LC-MS m/z (\%): 292 (100) [M+]; Anal. Calcd. for $\mathrm{C}_{10} \mathrm{H}_{14} \mathrm{FN}_{2} \mathrm{O}_{5} \mathrm{P}$ (\%): C, 41.10; H, 4.83; N, 9.59. Found: C, 41.03; $\mathrm{H}, 4.80 ; \mathrm{N}, 9.55$.

\section{Tetraethyl}

((phenylazanediyl) bis(methylene))diphosphoramidate (3f): Yield: 84\%; Brown solid; IR (ZnSe): 3345 (NH Aromatic), $1246(\mathrm{P}=\mathrm{O}), 922$ $\left(\mathrm{P}-\mathrm{O}-\mathrm{C}_{\text {aliphatic }}\right) \mathrm{cm}^{-1} ;{ }^{1} \mathrm{H}$ NMR $(500 \mathrm{MHz}$, DMSO- $\left.d_{6}\right): \delta 1.21-1.32\left(12 \mathrm{H}, \mathrm{m}, 4 \mathrm{CH}_{3}\right)$, $1.98(1 \mathrm{H}, \mathrm{s}, \mathrm{NH}), 4.46-4.54\left(8 \mathrm{H}, \mathrm{m}, 4 \mathrm{CH}_{2}\right)$, 4.76-4.84 (4H, m, 2- $\left.\mathrm{CH}_{2}\right), \quad 6.86-7.32$ (5H, m, ArH) ppm; ${ }^{13} \mathrm{C}$ NMR (125 MHz, DMSO- $\left.d_{6}\right): \delta 16.08,58.02,62.22,115.22$, 122.05, 129.44, $150.66 \mathrm{ppm} ;{ }^{31} \mathrm{P}$ NMR $\left(200 \mathrm{MHz}, \mathrm{DMSO}-d_{6}\right): \delta 2.824 \mathrm{ppm}$; LC-MS m/z(\%): 423 (100) [M+]; Anal. Calcd. for $\mathrm{C}_{16} \mathrm{H}_{31} \mathrm{~N}_{3} \mathrm{O}_{6} \mathrm{P}_{2}$ (\%): C, 45.39; H, 7.38; N, 9.92. Found: C, 45.35; H, 7.32; N, 9.85.

\section{Diethyl thiazol-2-ylphosphoram-} idate (3g): Yield: 87\%; Brown solid; IR (ZnSe): 3356 (NH Aromatic), 1206 $(\mathrm{P}=\mathrm{O}), 938\left(\mathrm{P}-\mathrm{O}-\mathrm{C}_{\text {aliphatic }}\right) \mathrm{cm}^{-1} ;{ }^{1} \mathrm{H} \mathrm{NMR}$ $\left(500 \mathrm{MHz}, \mathrm{DMSO}-d_{6}\right): \delta 1.28-1.32(6 \mathrm{H}$, $\left.\mathrm{m}, 2 \mathrm{CH}_{3}\right), 3.95(1 \mathrm{H}, \mathrm{s}, \mathrm{NH}), 4.46-4.54$ $\left(4 \mathrm{H}, \mathrm{m}, 2 \mathrm{CH}_{2}\right), 6.76-7.62(2 \mathrm{H}, \mathrm{m}, \mathrm{ArH})$ ppm; ${ }^{13} \mathrm{C}$ NMR (125 MHz, DMSO- $\left.d_{6}\right): \delta$ 16.36, 62.92, 115.21, 135.48, 169.82 ppm; ${ }^{31} \mathrm{P}$ NMR (200 MHz, DMSO- $d_{6}$ ): $\delta 2.821$ ppm; LC-MS m/z (\%): 236 (100) $[\mathrm{M}+]$; Anal. Calcd. for $\mathrm{C}_{7} \mathrm{H}_{13} \mathrm{~N}_{2} \mathrm{O}_{3} \mathrm{PS}(\%)$ : C, 35.59; H, 5.55; N, 11.86. Found: C, 35.53; $\mathrm{H}, 5.52 ; \mathrm{N}, 11.81$.

Diethyl (5-ethyl-1,3,4-thiadiazol-2-yl) phosphoramidate (3h): Yield: $86 \%$; Brown solid; IR (ZnSe): 3315 ( $\mathrm{NH}$ Aromatic), $1242(\mathrm{P}=\mathrm{O}), 944\left(\mathrm{P}-\mathrm{O}-\mathrm{C}_{\text {aliphatic }}\right) \mathrm{cm}^{-1} ;{ }^{1} \mathrm{H}$ NMR (500 MHz, DMSO- $\left.d_{6}\right): \delta 1.27-1.32$ $\left(6 \mathrm{H}, \mathrm{m}, 2 \mathrm{CH}_{3}\right), 1.34-1.37\left(3 \mathrm{H}, \mathrm{m}, \mathrm{CH}_{3}\right)$, 2.57-2.62 (2H, m, CH $), 4.05(1 \mathrm{H}, \mathrm{s}$, $\mathrm{NH}), 4.46-4.54\left(4 \mathrm{H}, \mathrm{m}, 2 \mathrm{CH}_{2}\right) \mathrm{ppm} ;{ }^{13} \mathrm{C}$ NMR (125 MHz, DMSO- $\left.d_{6}\right): \delta 11.56$, 16.52, 22.36, 62.96, 167.86, 174.14 ppm; ${ }^{31} \mathrm{P}$ NMR (200 MHz, DMSO-d $): \delta 2.842$ ppm; LC-MS m/z (\%): 265 (100) [M+]; Anal. Calcd. for $\mathrm{C}_{8} \mathrm{H}_{16} \mathrm{~N}_{3} \mathrm{O}_{3} \mathrm{PS}$ (\%): C, 36.22; H, 6.08; N, 15.84. Found: C, 36.17; H, 6.05; N, 15.75 .

\section{Diethyl benzo[d][1,3]diox-} ol-5-ylphosphoramidate (3i): Yield: 82\%; Brown solid; IR (ZnSe): 3352 (NH Aromatic), $1222(\mathrm{P}=\mathrm{O}), 953$ (P-O-C $\mathrm{Cliphatic})$ $\mathrm{cm}^{-1}$; ${ }^{1} \mathrm{H}$ NMR (500 MHz, DMSO-d 6 ): $\delta$ 1.27-1.33 $\left(6 \mathrm{H}, \mathrm{m}, 2 \mathrm{CH}_{3}\right), 4.01(1 \mathrm{H}, \mathrm{s}$, $\mathrm{NH}), 4.48-4.54\left(4 \mathrm{H}, \mathrm{m}, 2 \mathrm{CH}_{2}\right), 6.06-6.09$ $\left(2 \mathrm{H}, \mathrm{m}, \mathrm{OCH}_{2} \mathrm{O}\right), 6.12-6.65(3 \mathrm{H}, \mathrm{m}$, ArH) ppm; ${ }^{13} \mathrm{C}$ NMR (125 MHz, DMSO$\left.d_{6}\right): \delta 16.08,62.22,100.52,101.33,109.26$, 113.02, 132.88, 139.04, $149.12 \mathrm{ppm}$; ${ }^{31} \mathrm{P} \quad \mathrm{NMR} \quad\left(200 \mathrm{MHz}, \quad \mathrm{DMSO}-d_{6}\right)$ : $\delta 2.836 \mathrm{ppm} ; \mathrm{LC}-\mathrm{MS} \mathrm{m} / z$ (\%): 273 (100) $[\mathrm{M}+]$; Anal. Calcd. for $\mathrm{C}_{11} \mathrm{H}_{16} \mathrm{NO}_{5} \mathrm{P}(\%)$ : C, 48.36; H, 5.90; N, 5.13. Found: C, 48.33; H, 5.85; N, 5.05 .

\section{Diethyl (2-(1H-indol-3-yl)ethyl)} phosphoramidate (3j): Yield: $84 \%$; Brown solid; IR (ZnSe): 3344 (NH Aromatic), $1251(\mathrm{P}=\mathrm{O}), 958$ ( $\left.\mathrm{P}-\mathrm{O}-\mathrm{C}_{\text {aliphatic }}\right)$ $\mathrm{cm}^{-1}$; ${ }^{1} \mathrm{H}$ NMR (500 MHz, DMSO- $\left.d_{6}\right)$ : $\delta 1.28-1.34\left(6 \mathrm{H}, \mathrm{m}, 2 \mathrm{CH}_{3}\right), 2.04(1 \mathrm{H}, \mathrm{s}$, $\mathrm{NH}), 2.78-2.84\left(2 \mathrm{H}, \mathrm{m}, \mathrm{CH}_{2}\right), 2.92-2.94$ $\left(2 \mathrm{H}, \mathrm{m}, \mathrm{CH}_{2}\right), 4.47-4.52\left(4 \mathrm{H}, \mathrm{m}, 2 \mathrm{CH}_{2}\right)$, 7.16-7.42 (5H, m, ArH), $10.04(1 \mathrm{H}, \mathrm{s}$, Indole $\mathrm{NH}$ ) ppm; ${ }^{13} \mathrm{C} \mathrm{NMR} \mathrm{(125} \mathrm{MHz,}$ DMSO- $\left.d_{6}\right): \delta 16.05,31.02,43.95,62.32$, $111.23,114.26,118.98,119.84,122.08$, 124.54 ppm; ${ }^{31} \mathrm{P}$ NMR (200 MHz, DMSO$\left.d_{6}\right): \delta 10.252 \mathrm{ppm}$; LC-MS m/z (\%): 296 (100) $[\mathrm{M+}]$; Anal. Calcd. for $\mathrm{C}_{14} \mathrm{H}_{21} \mathrm{~N}_{2} \mathrm{O}_{3} \mathrm{P}$ (\%): C, 56.75; H, 7.14; N, 9.45. Found: C, $56.71 ; \mathrm{H}, 7.10 ; \mathrm{N}, 9.39$. 


\section{Diethyl (5-nitrothiazol-2-yl)phos-} phoramidate (3k): Yield: $80 \%$; Brown solid; IR (ZnSe): 3352 (NH Aromatic), $1216(\mathrm{P}=\mathrm{O}), 946\left(\mathrm{P}-\mathrm{O}-\mathrm{C}_{\text {aliphatic }}\right) \mathrm{cm}^{-1} ;{ }^{1} \mathrm{H}$ NMR (500 MHz, DMSO- $d_{6}$ ): $\delta 1.27-1.31$ $\left(6 \mathrm{H}, \mathrm{m}, 2 \mathrm{CH}_{3}\right), 4.01(1 \mathrm{H}, \mathrm{s}, \mathrm{NH}), 4.50-$ $4.54\left(4 \mathrm{H}, \mathrm{m}, 2 \mathrm{CH}_{2}\right), 8.62(1 \mathrm{H}, \mathrm{s}, \mathrm{ArH})$ ppm; ${ }^{13} \mathrm{C}$ NMR (125 MHz, DMSO- $\left.d_{6}\right)$ : $\delta$ 16.54, 62.32, 136.26, 147.38, 165.94 ppm; ${ }^{31} \mathrm{P}$ NMR (200 MHz, DMSO- $\left.d_{6}\right)$ : $\delta 2.812 \mathrm{ppm}$; LC-MS m/z (\%): 281 (100) $[\mathrm{M}+]$; Anal. Calcd. for $\mathrm{C}_{7} \mathrm{H}_{12} \mathrm{~N}_{3} \mathrm{O}_{5} \mathrm{PS}(\%)$ : C, 29.90; H, 4.30; N, 14.94. Found: C, 29.85; H, 4.26; N, 14.90 .

\section{Diethyl (2-(piperidin-2-yl)ethyl)} phosphoramidate (3l): Yield: 82\%; Brown solid; IR (ZnSe): 3362 (NH Aromatic), $1214(\mathrm{P}=\mathrm{O}), 953\left(\mathrm{P}-\mathrm{O}-\mathrm{C}_{\text {aliphatic }}\right)$ $\mathrm{cm}^{-1}$; ${ }^{1} \mathrm{H}$ NMR (500 MHz, DMSO- $d_{6}$ ): $\delta$ $1.28-1.35\left(6 \mathrm{H}, \mathrm{m}, 2 \mathrm{CH}_{3}\right), 1.56-1.64(8 \mathrm{H}$, $\left.\mathrm{m}, 4 \mathrm{CH}_{2}\right), 2.04(1 \mathrm{H}, \mathrm{s}, \mathrm{NH}), 2.66(1 \mathrm{H}, \mathrm{s}$, $\mathrm{CH}), 2.76-2.82\left(4 \mathrm{H}, \mathrm{m}, 2 \mathrm{CH}_{2}\right), 4.45-4.52$ $\left(4 \mathrm{H}, \mathrm{m}, 2 \mathrm{CH}_{2}\right), \mathrm{ppm} ;{ }^{13} \mathrm{C} \mathrm{NMR}(125 \mathrm{MHz}$, DMSO- $\left.d_{6}\right): \delta 16.28,23.32,26.85,32.82$, 35.06, 39.32, 47.02, 59.02, $62.22 \mathrm{ppm}$; ${ }^{31} \mathrm{P}$ NMR (200 MHz, DMSO- $\left.d_{6}\right): \delta$ $10.232 \mathrm{ppm}$; LC-MS m/z (\%): 264 (100) [M+]; Anal. Calcd. for $\mathrm{C}_{11} \mathrm{H}_{25} \mathrm{~N}_{2} \mathrm{O}_{3} \mathrm{P}(\%)$ : C, 49.99; H, 9.53; N, 10.60. Found: C, 49.95; H, 9.50; N, 10.54 .

\section{Results and Discussion}

At the onset of our investigation for the synthesis of phosphoramidate derivatives, 4-Chloro aniline and diethyl chlorophosphate were taken as model substrates to optimize the experimental conditions. Initially, 4-chloro aniline and diethyl chlorophosphate were heated at $80{ }^{\circ} \mathrm{C}$ in DMSO without any catalyst, but the reaction was unable to produce the product even after the prolonged heating for $48 \mathrm{~h}$ (Table 1, entry 1). Hence, we have traced the activity of various catalysts for
Diethyl (furan-2-ylmethyl)phosphoramidate (3m): Yield: $86 \%$; Brown solid; IR (ZnSe): 3348 (NH Aromatic), 1209 $(\mathrm{P}=\mathrm{O}), 968\left(\mathrm{P}-\mathrm{O}-\mathrm{C}_{\text {aliphatic }}\right) \mathrm{cm}^{-1} ;{ }^{1} \mathrm{H}$ NMR $\left(500 \mathrm{MHz}, \mathrm{DMSO}-d_{6}\right): \delta 1.22-1.32(6 \mathrm{H}$, $\left.\mathrm{m}, 2 \mathrm{CH}_{3}\right), 1.96(1 \mathrm{H}, \mathrm{s}, \mathrm{NH}), 3.72-3.76$ $\left(2 \mathrm{H}, \mathrm{m}, \mathrm{CH}_{2}\right), 4.49-4.54\left(4 \mathrm{H}, \mathrm{m}, 2 \mathrm{CH}_{2}\right)$, 6.46-7.62 (3H, m, ArH) ppm; ${ }^{13} \mathrm{C}$ NMR $\left(125 \mathrm{MHz}, \mathrm{DMSO}-d_{6}\right): \delta 16.22,35.01$, $62.12,110.26,110.48,142.54,148.82 \mathrm{ppm}$; ${ }^{31} \mathrm{P}$ NMR $\left(200 \mathrm{MHz}, \mathrm{DMSO}-d_{6}\right): \delta 7.824$ ppm; LC-MS m/z (\%): 233 (100) [M+]; Anal. Calcd. for $\mathrm{C}_{9} \mathrm{H}_{16} \mathrm{NO}_{4} \mathrm{P}(\%)$ : C, 46.35; H, 6.92; N, 6.01. Found: C, 46.30; H, 6.88; N, 5.94 .

Diethyl 1H-benzo[d]imidazol-1-ylphosphonate (3n): Yield: 84\%; Brown solid; IR (ZnSe): 3356 (NH Aromatic), $1198(\mathrm{P}=\mathrm{O}), 959$ (P-O-C $\left.\mathrm{C}_{\text {aliphatic }}\right)$ $\mathrm{cm}^{-1}$; ${ }^{1} \mathrm{H}$ NMR (500 MHz, DMSO- $d_{6}$ ): $\delta$ 1.26-1.34 $\left(6 \mathrm{H}, \mathrm{m}, 2 \mathrm{CH}_{3}\right), 4.50-4.53(4 \mathrm{H}$, $\left.\mathrm{m}, 2 \mathrm{CH}_{2}\right), 7.26-7.62(4 \mathrm{H}, \mathrm{m}, \mathrm{ArH}), 8.18$ $(1 \mathrm{H}, \mathrm{s}, \mathrm{N}=\mathrm{CH}-\mathrm{N}) \mathrm{ppm} ;{ }^{13} \mathrm{C}$ NMR $(125$ MHz, DMSO- $\left.d_{6}\right): \delta 16.22,60.12,115.26$, $124.08,137.84,139.05,142.25 \mathrm{ppm} ;{ }^{31} \mathrm{P}$ NMR $\left(200 \mathrm{MHz}\right.$, DMSO- $\left.d_{6}\right): \delta-6.724$ ppm; LC-MS m/z (\%): 254 (100) [M+]; Anal. Calcd. for $\mathrm{C}_{11} \mathrm{H}_{15} \mathrm{~N}_{2} \mathrm{O}_{3} \mathrm{P}$ (\%): C, 51.97; H, 5.95; N, 11.02. Found: C, 51.93; $\mathrm{H}, 5.91 ; \mathrm{N}, 10.96$.

the synthesis of Diethyl(4-chlorophenyl) phosphoramidate (3a). The catalytic effect of such inorganic and organic bases (Table 1, entries 2-11) afforded the products with low yield, where $\mathrm{K}_{2} \mathrm{CO}_{3}$ only afforded maximum product yields in $8 \mathrm{~h}$ of reaction time (Table 1, entries 12-14). In the catalyst optimization studies with 2 , 5 and $10 \mathrm{~mol} \%$ of $\mathrm{K}_{2} \mathrm{CO}_{3}$, we obtained the yields were 68, 94 and 94 respectively (Table 1, Entries 12-14). Therefore, $5 \mathrm{~mol} \%$ of $\mathrm{K}_{2} \mathrm{CO}_{3}$ was sufficient for completion of 
Influence of various catalysts on the synthesis of compound 4 a at $80{ }^{\circ} \mathrm{C}$

\begin{tabular}{|c|c|c|c|c|}
\hline Entry & Catalyst & Catalyst (mol \%) & Time (h) & Yield (\%) \\
\hline 1 & None & - & 48 & NR \\
\hline 2 & $\mathrm{Cs}_{2} \mathrm{CO}_{3}$ & 5 & 10 & 65 \\
\hline 3 & $\mathrm{Na}_{2} \mathrm{CO}_{3}$ & 5 & 12 & 45 \\
\hline 4 & $\mathrm{NaOH}$ & 5 & 24 & NR \\
\hline 5 & $t-\mathrm{BuOH}$ & 5 & 10 & 42 \\
\hline 6 & $\mathrm{NaHCO}_{3}$ & 5 & 10 & 38 \\
\hline 7 & $\mathrm{~K}_{3} \mathrm{PO}_{4} \cdot 3 \mathrm{H}_{2} \mathrm{O}$ & 5 & 24 & NR \\
\hline 8 & $\mathrm{AcOK}$ & 5 & 24 & NR \\
\hline 9 & DBU & 5 & 14 & Trace \\
\hline 10 & $\mathrm{Et}_{3} \mathrm{~N}$ & 5 & 10 & 24 \\
\hline 11 & Pyridine & 5 & 10 & 15 \\
\hline 12 & $\mathrm{~K}_{2} \mathrm{CO}_{3}$ & 2 & 8 & 68 \\
\hline 13 & $\mathrm{~K}_{2} \mathrm{CO}_{3}$ & 5 & 8 & $94,89,82$ \\
\hline 14 & $\mathrm{~K}_{2} \mathrm{CO}_{3}$ & 10 & 8 & 94 \\
\hline
\end{tabular}

Table 2

the reaction and excess amount of catalyst did not increase the yields and the reusability of the catalyst has not also been observed with the mark of satisfaction.

Then several solvents, such as DMF, 1,4-dioxane, acetone, MeCN, THF, $\mathrm{CH}_{3} \mathrm{NO}_{2}, \mathrm{CH}_{3} \mathrm{CH}_{2} \mathrm{OH}$, and dimethylsulfoxide were screened in the presence of 5 mol\% of $\mathrm{K}_{2} \mathrm{CO}_{3}$ at $80{ }^{\circ} \mathrm{C}$ (Table 2, entries $1-8)$, and the results showed that dimethylsulfoxide (DMSO) was the best choice.
Effect of various solvents on the synthesis of compound $4 \mathrm{a}$

\begin{tabular}{c|c|c|c} 
Entry & Solvent & Time (h) & Yield (\%) \\
\hline 1 & DMF & 8 & 74 \\
\hline 2 & 1,4-dioxane & 8 & 72 \\
\hline 3 & Acetone & 8 & 68 \\
\hline 4 & MeCN & 8 & 75 \\
\hline 5 & THF & 8 & 82 \\
\hline 6 & $\mathrm{CH}_{3} \mathrm{NO}_{2}$ & 8 & 42 \\
\hline 7 & $\mathrm{CH}_{3} \mathrm{CH}_{2} \mathrm{OH}$ & 8 & 84 \\
\hline 8 & $\mathrm{DMSO}$ & 8 & 94
\end{tabular}

\section{Conclusions}

We have been successful in accomplishing a new synthetic protocol for the construction of phosphoramidates scaffold under sustainable condition applying $\mathrm{K}_{2} \mathrm{CO}_{3}$ catalysis. Developed synthetic protocol offers various advantages like op- erational simplicity, low catalyst loading, an extensive substrate scope, and a high product yield. The use of DMSO as the reaction medium and application of $\mathrm{K}_{2} \mathrm{CO}_{3}$ catalyst make this protocol truly a practical one for synthetic chemistry.

\section{Acknowledgements}

Acknowledgements: We thank Prof. C. Devendranath Reddy, Department of Chemistry, S. V. University, Tirupati for his helpful discussions and Science and Engineering Research Board (SERB),
New Delhi - 110070 India for providing financial assistance through a research project grant F. No.: SB/S1/OC-96/2013, Dt: 05-11-2014. 


\section{References}

1. Egron D, Imbach JL, Gosselin G, Aubertin AM, Perigaud C. S-Acyl-2-thioethyl Phosphoramidate Diester Derivatives as Mononucleotide Prodrugs. J Med Chem. 2003;46(21):4564-71. DOI:10.1021/jm0308444.

2. Denmark SE, Chung WJ. Lewis Base Activation of Lewis Acids: Catalytic Enantioselective Glycolate Aldol Reactions. Angew Chem Int Ed. 2008;47(10):1890-2. DOI:10.1002/anie.200705499.

3. Yadav LDS, Srivastava VP, Patel R. The first application of the Baylis-Hillman reaction in azetidine chemistry: a convenient synthesis of azetidine-3-carbonitriles/ carboxylates. Tetrahedron Lett. 2008;49(39):5652-4. DOI:10.1016/j.tetlet.2008.07.069.

4. Minami T, Ogata M, Hirao I, Tanaka M, Agawa T. Synthesis of a 3,1-Benzoxazin-4-one, 2,4(1H,3H)-Quinolinediones, and 2,4(1H,3H)-Quinazolinediones from the Reaction of Phosphoryl-Stabilized Anions Containing no a-Hydrogen Atoms with Isatoic Anhydride. Synthesis. 1982;1982(3):231-3. DOI:10.1055/s-1982-29760.

5. Radkiewicz JL, McAllister MA, Goldstein E, Houk KN. A Theoretical Investigation of Phosphonamidates and Sulfonamides as Protease Transition State Isosteres. J Org Chem. 1998;63(5):1419-28. DOI:10.1021/jo971425f.

6. Roberts WP, Tate ME, Kerr A. Agrocin 84 is a 6-N-phosphoramidate of an adenine nucleotide analogue. Nature. 1977;265:379-81. DOI:10.1038/265379a0.

7. Phillips DR, Uramoto M, Isono K, McCloskey JA. Structure of the antifungal nucleotide antibiotic phosmidosine. J Org Chem. 1993;58(4):854-9. DOI:10.1021/ jo00056a017.

8. Cho A, Zhang L, Xu J, Lee R, Butler T, Metobo S, Aktoudianakis V, Lew W, Ye H, Clarke M, Doerffler E, Byun D, Wang T, Babusis D, Carey AC, German P, Sauer D, Zhong W, Rossi S, Fenaux M, Mc Hutchison JG, Perry J, Feng J, Ray AS, Kim CU. Discovery of the First C-Nucleoside HCV Polymerase Inhibitor (GS-6620) with Demonstrated Antiviral Response in HCV Infected Patients. J Med Chem. 2014;57:1812-25. DOI:10.1021/jm400201a.

9. Charlton M, Gane E, Manns MP, Brown RS, Curry MP, Kwo PY, Fontana RJ, Gilroy R, Teperman L, Muir AJ, McHutchison JG, Symonds WT, Brainard D, Kirby B, Dvory-Sobol H, Denning J, Arterburn S, Samuel D, Forns X, Terrault NA. Sofosbuvir and Ribavirin for Treatment of Compensated Recurrent Hepatitis C Virus Infection After Liver Transplantation. Gastroenterology. 2015;148(1):108-17. DOI:10.1053/j. gastro.2014.10.001.

10. World Health Organization. Proposed INN: List 111. WHO Drug Information [Internet]. 2014[cited 2017 Jun 14];28(2):211-94. Available from: http://www.who.int/ medicines/publications/druginformation/PL_111.pdf.

11. Adelfinskaya O, Herdewijn P. Amino Acid Phosphoramidate Nucleotides as Alternative Substrates for HIV-1 Reverse Transcriptase. Angew Chem Int Ed. 2007;46(23):4356-8. DOI:10.1002/anie.200605016.

12. Gao X, Tang Z, Lu M, Liu H, Jiang Y, Zhao Y, Cai Z. Suppression of matrix ions by N-phosphorylation labeling using matrix-assisted laser desorption-ionization timeof-flight mass spectrometry. Chem Commun. 2012;48(82):10198-200. DOI:10.1039/ c2cc36091h. 
13. Overtveldt MV, Heugebaeurt TSA, Verstraeten I, Geelen D, Stevens CV. Phosphonamide pyrabactin analogues as abscisic acid agonists. Org Biomol Chem. 2015;13(18):5260-4. DOI:10.1039/C5OB00137D.

14. Bakibaev AA, Yagovkin AY, Vostretsov SN. Methods of synthesis of nitrogencontaining heterocycles using ureas and related compounds. Russ Chem Rev. 1998;67(4): 295-314. DOI:10.1070/RC1998v067n04ABEH000295.

15. Milen M, Abranyi-Balogh P, Balogh G, Drahos L, Keglevich GA. Study on the Phosphorylation of Indole, Imidazole, Carbazole, and Phenothiazine Derivatives. Phosphorus, Sulfur Silicon Relat Elem. 2012;187(9):1091-100. DOI:10.1080/10426507.2 012.668986.

16. Davis TA, Wilt JC, Johnston JN. Bifunctional Asymmetric Catalysis: Amplification of Brønsted Basicity Can Orthogonally Increase the Reactivity of a Chiral Brønsted Acid. J Am Chem Soc. 2010;132(9):2880-2. DOI:10.1021/ja908814h.

17. Xiang DF, Bigley AN, Ren Z, Xue H, Hull KG, Romo D, Raushel FM. Interrogation of the Substrate Profile and Catalytic Properties of the Phosphotriesterase from Sphingobium sp. Strain TCM1: An Enzyme Capable of Hydrolyzing Organophosphate Flame Retardants and Plasticizers. Biochemistry. 2015;54(51):7539-49. DOI:0.1021/acs.biochem.5b01144.

\section{Cite this article as (как цитировать эту статью)}

Ramana PV, Krishna BS, Reddy NB, Sravaya G, Zyryanov GV, Reddy C S. Potassium carbonate supported efficient synthesis of new diethyl arylphosphoramidates. Chimica Techno Acta. 2017;4(2):148-156. DOI:10.15826/chimtech.2017.4.2.030. 